ARTIFICIAL SATELLITES, Vol. 43, No. $4-2008$
DOI: $10.2478 / \mathrm{v} 10018-009-0012-9$

\title{
INSIGHT INTO THE FOURIER TRANSFORM BAND PASS FILTERING TECHNIQUE
}

\author{
Waldemar Popiński \\ Space Research Centre PAS \\ e-mail:w.popinski@stat.gov.pl
}

\begin{abstract}
In this work theoretical foundations of the Fourier Transform Band Pass Filter (FTBPF) technique are developed which show that such procedure is applicable to extraction of irregular monochromatic oscillations with time-varying amplitudes and phases from the analyzed signal data. Considerations concerning the statistical error of the FTBPF coherence between two filtered oscillations are included. Further certain method of the filter pass-band width parameter determination is proposed and relation between applying the FTBPF to realvalued signals and forming the so called complex-valued analytic oscillations based on the Hilbert transform is indicated.
\end{abstract}

Keywords: Fourier Transform Band Pass Filter, transmittance function, pass-band width, irregular oscillation, Hilbert Transform

\section{INTRODUCTION}

The filtering technique based on the Fourier Transform and appropriate transmittance functions has been applied in the spectral analysis of time series for more than 30 years now Brillinger (1975), Koopmans (1974). It is of interest to scientists working in the domain of geophysics Forbes (1988), Pan (1998), geodesy Kosek (1995), Popiński and Kosek (1995) and signal processing Brillinger (1975), Koopmans (1974), Pan (2001), Speed (1985), since it enables analyzing stationary time series in chosen frequency bands. It can be also used in digital image processing Hoggar (2006), Press et al. (1992) and spectral analysis of twodimensional maps of sea surface topography Popiński and Kosek (1999). Certain theoretical as well as numerical properties of the considered filtration technique were already examined and described in time series analysis textbooks Brillinger (1975), Koopmans (1974) and articles concerning its applications Forbes (1988), Pan (1998), but they were related rather to the case of stationary time series analysis and errors or effects like the Gibbs phenomenon connected with numerical implementation of such a technique Forbes (1988), Pan (2001).

Since there is no analytical model of polar motion available yet such tool is particularly needed in the investigation of oscillations occurring in Earth rotation parameters data (time series of pole coordinates and length-of-day) and in examining the influence of relevant angular momentum excitation functions on them. In consequence, there are some works of several authors on its application in this domain of research Kołaczek (1992), Kołaczek and Kosek (1993), Kosek (1995, 2004), Kosek et al. (1995), Kosek and Kaczkowski (1994), Kosek and Popiński (1999), Nastula et al. (1993), Popiński and Kosek (1995, 2000). 
The present work is supposed to be a continuation of author's previous publications Popiński and Kosek (1995), Popiński (1997) on theoretical, statistical, as well as numerical properties of Fourier Transform Band Pass Filter (FTBPF) technique. This time the work deals with the problem whether the considered filtering procedure can give us an image of temporal evolution of irregular oscillations with variable amplitudes and phases, present in the analyzed time series (section 2). Investigations on a related subject using ideas based on the Fourier Transform, complex demodulation and tapering were already published by Hasan (1983) and Park (1992).

Having filtered oscillations from two time series using the FTBPF one can compute their correlation coefficient i.e. their coherence in the chosen filter frequency band Koopmans (1974). The problem of FTBPF coherence statistical significance is dealt with in section 3 by deriving an estimate of the coherence statistical error and indicating its basic properties Koopmans (1974).

Since choosing proper spectral pass-band width parameter of the filter is a crucial matter for precise filtering of the examined oscillations from the available time series Popinski and Kosek (1995), a method of selecting pass-band width value is proposed in section 4 and hints for its numerical implementation are included.

In section 5 relationship between filtering oscillations from real-valued signals using the FTBPF and forming complex-valued analytic oscillations form real-valued ones with appliance of the Hilbert transform as their imaginary component is indicated Gasquet and Witomski (1999).

\section{THEORETICAL PROPERTIES OF THE FTBPF}

The FTBPF is used to filter a narrow-band oscillation with chosen central frequency $\omega_{c}$ from the analyzed signal represented by a complex-valued function $x(t)=\operatorname{Re}[x(t)]+i \operatorname{Im}[x(t)]$ of continuous time argument, satisfying $x \in L^{2}(R)$. According to the definition of this filter such an oscillation is given by the formula Koopmans (1974):

$$
o_{x}\left(\omega_{c}, t\right)=C F T^{-1}\left[A\left(\omega_{c}, \omega\right) C F T[x(s)]\right],
$$

where the CFT operator denotes the Continuous Fourier Transform of the function $x(s)$,

$$
C F T[x(s)]=\widehat{x}(\omega)=\int_{-\infty}^{+\infty} x(s) \exp (-i \omega s) d s,
$$

and $A\left(\omega_{c}, \omega\right)$ is a real-valued transmittance function of the filter, which is different from zero only in the neighborhood of the central frequency $\omega_{c}$ and satisfies the conditions $0 \leq A\left(\omega_{c}, \omega\right) \leq 1, A\left(\omega_{c}, \omega_{c}\right)=1$. As an example of the transmittance function one can choose the simple boxcar function Speed (1985)

$$
A_{R}\left(\omega_{c}, \omega\right)=\left\{\begin{array}{lll}
1 & \text { for } & \left|\omega-\omega_{c}\right| \leq 2 \pi \lambda, \\
0 & \text { for } & \left|\omega-\omega_{c}\right|>2 \pi \lambda,
\end{array}\right.
$$

but the oscillation can be filtered more precisely if we use smoother transmittance functions like the parabolic one Popiński and Kosek (1995)

$$
A_{P}\left(\omega_{c}, \omega\right)=\left\{\begin{array}{ccc}
1-\left(\frac{\omega}{2 \pi \lambda}\right)^{2} & \text { for } & \left|\omega-\omega_{c}\right| \leq 2 \pi \lambda, \\
0 & \text { for } & \left|\omega-\omega_{c}\right|>2 \pi \lambda,
\end{array}\right.
$$

or the cosine-bell function Popiński and Kosek (1995) 


$$
A_{B}\left(\omega_{c}, \omega\right)=\left\{\begin{array}{ccc}
\frac{1}{2}\left[1+\cos \left(\frac{\omega-\omega_{c}}{2 \lambda}\right)\right] & \text { for } & \left|\omega-\omega_{c}\right| \leq 2 \pi \lambda, \\
0 & \text { for } & \left|\omega-\omega_{c}\right|>2 \pi \lambda,
\end{array}\right.
$$

where $\lambda>0$ is the filter pass-band width parameter.

The FTBPF filtration technique with the use of such transmittance functions is directly related to the Harmonic Wavelet Transform (HWT) Newland (1993, 1994, 1998). In the HWT the coefficients of the analyzed signal $x(t)$, corresponding to the selected harmonic wavelet function $h \in L^{2}(R)$ with $C F T[h(s)]=\hat{h}\left(\omega_{c}, \omega\right)=A\left(\omega_{c}, \omega\right)$, are determined as follows

$$
H\left(\omega_{c}, \tau\right)=\int_{-\infty}^{+\infty} x(t) \bar{h}(t-\tau) d t=\frac{1}{2 \pi} \int_{-\infty}^{+\infty} \bar{x}(\omega) \overline{\widehat{h}}\left(\omega_{c}, \omega\right) \exp (i \tau \omega) d \omega=o_{x}\left(\omega_{c}, \tau\right),
$$

where $\tau \in R$ is the translation parameter, and the second equality follows easily from the classical Plancherel identity for the CFT, in which over-bar denotes complex conjugation and $i=\sqrt{-1}$ Bremaud (2002). The same Plancherel identity together with definition (1) or (2) gives us the following formula for the energy of the filtered oscillation

$$
\int_{-\infty}^{\infty}\left|o_{x}\left(\omega_{c}, s\right)\right|^{2} d s=\frac{1}{2 \pi} \int_{-\infty}^{\infty}\left|A\left(\omega_{c}, \omega\right)\right|^{2}|\widehat{x}(\omega)|^{2} d \omega \leq \frac{1}{2 \pi} \int_{-\infty}^{\infty}|\widehat{x}(\omega)|^{2} d \omega=\int_{-\infty}^{\infty}|x(s)|^{2} d s,
$$

since $\left|A\left(\omega_{c}, \omega\right)\right| \leq 1$ as assumed earlier. The last inequality together with linearity of the CFT assures that filtering of oscillations using the FTBPF technique is a linear operation continuous in the $L^{2}(R)$ norm.

Observe now that for sufficiently regular transmittance functions like the ones mentioned above we immediately obtain from the definition of the inverse CFT Bremaud (2002)

$$
h(t)=\frac{1}{2 \pi} \int_{-\infty}^{+\infty} \widehat{h}\left(\omega_{c}, \omega\right) \exp (i t \omega) d \omega=\frac{1}{2 \pi} \int_{-2 \pi \lambda}^{2 \pi \lambda} \hat{h}(0, v) \exp \left(i t\left(v+\omega_{c}\right)\right) d v=\exp \left(i t \omega_{c}\right) h_{0}(t),
$$

where $h_{0}(t)$ is the harmonic wavelet function with $\widehat{h}_{0}(\omega)=\hat{h}(0, \omega)=A(0, \omega)$. Simple analytical integration shows that the harmonic wavelet functions $h_{R 0}(t), h_{P 0}(t), h_{B 0}(t)$ with their CFT given by $A_{R}(0, \omega), A_{P}(0, \omega), A_{B}(0, \omega)$, respectively, are given by the corresponding formulae:

$$
\begin{aligned}
& h_{R 0}(t)=\frac{\sin (2 \pi \lambda t)}{\pi t} \quad \text { for } t \neq 0, h_{R 0}(0)=2 \lambda, \\
& h_{P 0}(t)=\frac{1}{2 \lambda^{2}(\pi t)^{3}}[\sin (2 \pi \lambda t)-2 \pi \lambda t \cos (2 \pi \lambda t)] \text { for } t \neq 0, h_{P 0}(0)=4 \lambda / 3, \\
& h_{B 0}(t)=\frac{-\sin (2 \pi \lambda t)}{2 \pi t(2 \lambda t+1)(2 \lambda t-1)} \text { for } t \neq 0, h_{B 0}(0)=\lambda, h_{B 0}( \pm 1 / 2 \lambda)=\lambda / 2 .
\end{aligned}
$$

The graphs of the chosen harmonic wavelet CFTs (FTBPF transmittance functions) are presented in Fig. 1 and the graphs of the wavelet functions themselves in Fig. 2.

One can clearly see from the above formulae that the functions $h_{R 0}(t), h_{P 0}(t), h_{B 0}(t)$ decrease to zero asymptotically as fast as $|t|^{-1},|t|^{-2},|t|^{-3}$, respectively, as $|t|$ tends to infinity. Putting $h(t)=\exp \left(i \omega_{c} t\right) h_{0}(t)$ in the definition (2) yields the equality

$$
H\left(\omega_{c}, \tau\right)=\int_{-\infty}^{+\infty} x(t) \bar{h}(t-\tau) d t=\int_{-\infty}^{+\infty} x(t) \bar{h}_{0}(t-\tau) \exp \left(-i \omega_{c}(t-\tau)\right) d t=C\left(\omega_{c}, \tau\right) \exp \left(i \omega_{c} \tau\right)
$$


where $C\left(\omega_{c}, \tau\right)=\int_{-\infty}^{+\infty} x(t) \bar{h}_{0}(t-\tau) \exp \left(-i \omega_{c} t\right) d t$

From the above formula we see that the FTBPF or equivalently the HWT with $\hat{h}\left(\omega_{c}, \omega\right)=A\left(\omega_{c}, \omega\right)$ enables obtaining information on temporal variations of amplitude and phase of irregular monochromatic oscillations of the form $o(t)=a(t) \exp \left(i \omega_{c} t+i \varphi(t)\right)$ present in the analyzed signal. Such information is contained in the modules $\left|C\left(\omega_{c}, \tau\right)\right|$ and phases $\psi\left(\omega_{c}, \tau\right)=\arctan \left(\operatorname{Im}\left[C\left(\omega_{c}, \tau\right)\right] / \operatorname{Re}\left[C\left(\omega_{c}, \tau\right)\right]\right)$ of the complex-valued function $C\left(\omega_{c}, \tau\right)=H\left(\omega_{c}, \tau\right) \exp \left(-i \omega_{c} \tau\right)$, since then we have $C\left(\omega_{c}, \tau\right)=\int_{-\infty}^{+\infty} a(t) \exp (i \varphi(t)) \bar{h}_{0}(t-\tau) d t$.

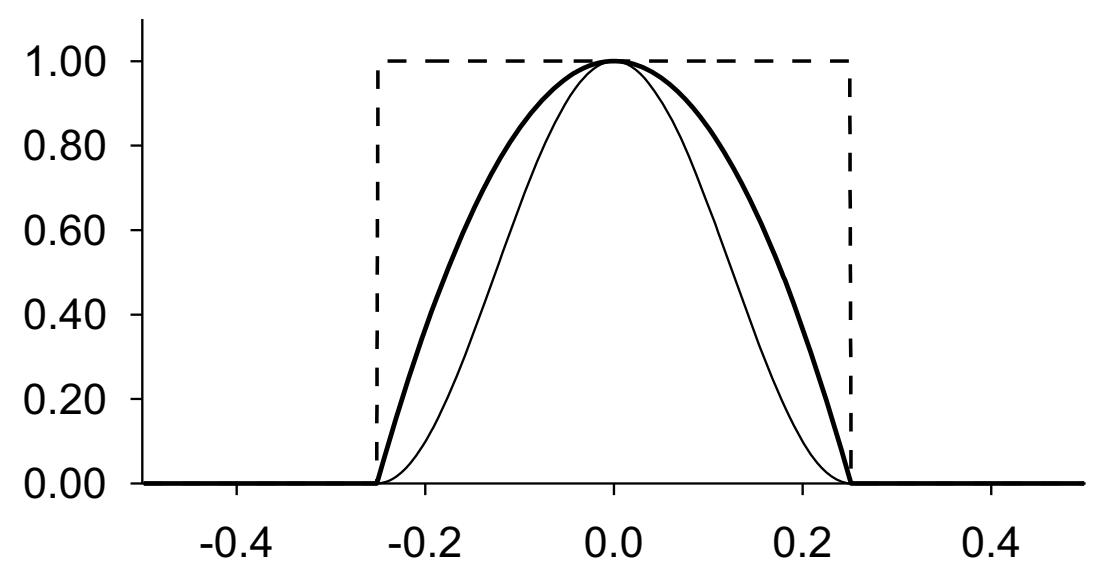

Fig. 1. The FTBPF boxcar ( $\lambda=0.04$, dashed line), parabolic ( $\lambda=0.04$, heavy line), and cosine-bell ( $\lambda=0.04$, thin line) transmittance functions (the harmonic wavelet CFTs).

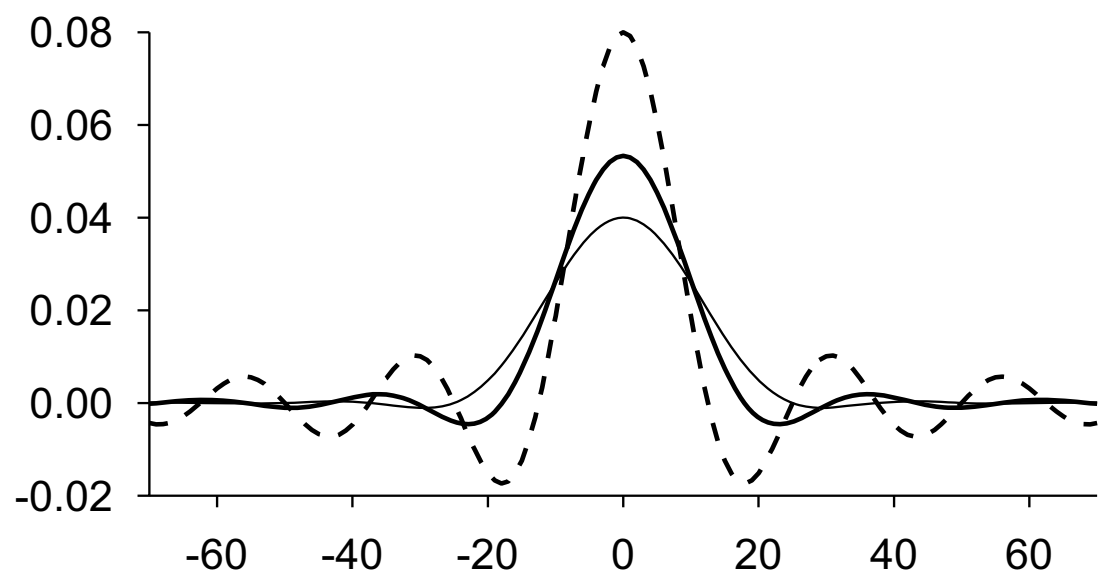

Fig. 2. The harmonic wavelet functions corresponding to the boxcar $(\lambda=0.04$, dashed line $)$, parabolic $(\lambda=0.04$, heavy line $)$, and cosine-bell ( $\lambda=0.04$, thin line) CFTs.

Moreover, the imposed normalizing condition $\widehat{h}_{0}(0)=\hat{h}(0,0)=A(0,0)=1$ assures that for monochromatic oscillations with constant amplitude and phase $o_{c}(t)=a \exp \left(i \omega_{c} t+i \varphi\right)$ calculating the HWT coefficients we simply reproduce the original oscillation. Indeed, analogously as in formula (3) we obtain 


$$
\begin{aligned}
H_{c}\left(\omega_{c}, \tau\right) & =\int_{-\infty}^{+\infty} o_{c}(t) \bar{h}(t-\tau) d t=a \exp (i \varphi) \int_{-\infty}^{+\infty} \exp \left(i \omega_{c} t\right) \bar{h}_{0}(t-\tau) \exp \left(-i \omega_{c}(t-\tau)\right) d t \\
& =a \exp \left(i \omega_{c} \tau+i \varphi\right) \int_{-\infty}^{+\infty} \bar{h}_{0}(t-\tau) d t=o_{c}(\tau) \int_{-\infty}^{+\infty} \bar{h}_{0}(s) d s=o_{c}(\tau) \overline{\widehat{h}}_{0}(0)=o_{c}(\tau) .
\end{aligned}
$$

For oscillations $o_{0}(t)=a \exp \left(i \omega_{0} t+i \varphi\right)$ with $\omega_{0} \neq \omega_{c}$ analogous calculation shows that $H_{0}\left(\omega_{c}, \tau\right)=o_{0}(\tau) A\left(0, \omega_{c}-\omega_{0}\right)$. This means that depending on the transmittance function the FTBPF passes only monochromatic oscillations with $\left|\omega_{0}-\omega_{c}\right| \leq 2 \pi \lambda$ but can also attenuate those with $\omega_{0} \neq \omega_{c}$. Furthermore, the described filtration technique enables analyzing oscillations with prograde $\left(\omega_{c}>0\right)$ and retrograde $\left(\omega_{c}<0\right)$ polarization in the case of complex-valued signals Kulesh et al. (2008).

In practice only time series of the analyzed signal observation values at discrete time moments $x(n), n=0,1, \ldots, N-1$, is available and then we can compute its Discrete Fourier Transform (DFT)

$$
\widetilde{x}(v)=\sum_{n=0}^{N-1} x(n) \exp (-i 2 \pi m / N),
$$

for $v=-N / 2+1,-N / 2+2, \ldots, N / 2$, and even values of $N$. If we approximate the frequency domain integral in (2) by summation with step $2 \pi / N$, and replace the values $\bar{x}(2 \pi \nu / N)$ by $\widetilde{x}(v)$ we obtain its discretized version

$$
H\left(\omega_{c}, \tau\right)=o_{x}\left(\omega_{c}, \tau\right) \cong \breve{o}_{x}\left(\omega_{c}, \tau\right)=\frac{1}{N} \sum_{v=-N / 2+1}^{N / 2} \widetilde{x}(v) \overline{\bar{h}}\left(\omega_{c}, 2 \pi v / N\right) \exp (i 2 \pi v \tau / N),
$$

and its computation for $\tau=0,1, \ldots, N-1$, can be performed using the inverse DFT. In consequence, for calculating the approximate values of the HWT coefficients or equivalently the approximate FTBPF oscillation values one can use the Fast Fourier Transform procedures Press et al. (1992), Singleton (1969). Of course, applying the DFT definition in (4) yields analogously as in (2) alternative time domain formula for the filtered oscillation values or the HWT coefficients

$$
\breve{o}_{x}\left(\omega_{c}, \tau\right)=\frac{1}{N} \sum_{n=0}^{N-1} x(n) \sum_{v=-N / 2+1}^{N / 2} \overline{\widehat{h}}\left(\omega_{c}, 2 \pi v / N\right) \exp (i 2 \pi v(\tau-n) / N) .
$$

The approximate values of the HWT coefficients determined in accordance with formula (4) allow us to estimate how strongly the analyzed signal is correlated with succeeding time domain translations $h(t-\tau), \tau=0,1, \ldots, N-1$, of the wavelet function $h$. Thus, the computed time series $H\left(\omega_{c}, \tau\right), \quad \tau=0,1, \ldots, N-1$, characterizes possibly non-stationary temporal variations of oscillations forming the analyzed signal, which have frequencies in the range $\left(\omega_{c}-2 \pi \lambda, \omega_{c}+2 \pi \lambda\right)$, where $-\pi<\omega_{c}-2 \pi \lambda<\omega_{c}<\omega_{c}+2 \pi \lambda<\pi$, since we deal with time series data with sampling interval $\Delta t=1$ Koopmans (1974). This important property does not follow directly from the formula (1) defining the band pass filtration technique considered here.

For calculation of correlation coefficients of oscillations filtered from time series data by the FTBPF it is also important that the mean values of such oscillations are always zero. Indeed, by (4) we have

$$
\sum_{\tau=0}^{N-1} \breve{o}_{x}\left(\omega_{c}, \tau\right)=\frac{1}{N} \sum_{v=-N / 2+1}^{N / 2} \tilde{x}(v) \overline{\bar{h}}\left(\omega_{c}, 2 \pi v / N\right) \sum_{\tau=0}^{N-1} \exp (i 2 \pi v \tau / N)
$$




$$
=\frac{1}{N} \sum_{v=-N / 2+1}^{N / 2} \tilde{x}(v) \overline{\widehat{h}}\left(\omega_{c}, 2 \pi v / N\right) \frac{1-\exp (i 2 \pi v)}{1-\exp (i 2 \pi v / N)}=0
$$

since $\exp (i 2 \pi v)=1$ for $v=-N / 2+1,-N / 2+2, \ldots, N / 2$. Furthermore, applying the Schwartz inequality Bremaud (2002) to (4) for $\tau=0,1, \ldots, N-1$, and summing with respect to the $\tau$ index yields

$$
\sum_{\tau=0}^{N-1}\left|\breve{o}_{x}\left(\omega_{c}, \tau\right)\right|^{2} \leq \frac{1}{N} \sum_{v=-N / 2+1}^{N / 2}|\widetilde{x}(v)|^{2} \sum_{v=-N / 2+1}^{N / 2}\left|\widehat{h}\left(\omega_{c}, 2 \pi v / N\right)\right|^{2}=\sum_{n=0}^{N-1}|x(n)|^{2} \sum_{v=-N / 2+1}^{N / 2}\left|\widehat{h}\left(\omega_{c}, 2 \pi v / N\right)\right|^{2},
$$

since the classical identity $\frac{1}{N} \sum_{v=-N / 2+1}^{N / 2}|\widetilde{x}(v)|^{2}=\sum_{n=0}^{N-1}|x(n)|^{2}$ holds Gasquet and Witomski (1999), which is also derived in the next section. Hence, we see that computing the vector of the approximate oscillation values $\breve{o}_{x}\left(\omega_{c}, \tau\right), \tau=0,1, \ldots, N-1$, is a linear operation continuous in the euclidean norm and simultaneously we obtain an estimate of the filtered oscillation energy.

\section{FTBPF COHERENCE AND ITS STATISTICAL ERROR}

Coherence is recognized as a measure of similarity of two signals in a chosen frequency band Koopmans (1974). For example in research on polar motion excitation one often compares the oscillations filtered from geodetic excitation functions determined from polar coordinates data with analogous oscillations filtered from geophysical excitation data like the equatorial component of atmospheric angular momentum Kosek and Popiński (1999), Popiński and Kosek (2000). If we denote oscillations filtered using the same FTBPF with central frequency $\omega_{c}$ from two time series $x(n), y(n), n=0,1, \ldots, N-1$, (equation (4)) by $\breve{o}_{x}\left(\omega_{c}, \tau\right)$, $\breve{o}_{y}\left(\omega_{c}, \tau\right), \tau=0,1, \ldots, N-1$, respectively, then their complex-valued coherence is given by the formula Koopmans (1974)

$$
\gamma_{x y}\left(\omega_{c}\right)=\frac{S_{x y}\left(\omega_{c}\right)}{\sqrt{S_{x x}\left(\omega_{c}\right) S_{y y}\left(\omega_{c}\right)}}
$$

where

$$
\begin{gathered}
S_{x y}\left(\omega_{c}\right)=\sum_{\tau=0}^{N-1} \breve{o}_{x}\left(\omega_{c}, \tau\right) \overline{\bar{o}}_{y}\left(\omega_{c}, \tau\right) \\
S_{x x}\left(\omega_{c}\right)=\sum_{\tau=0}^{N-1} \breve{o}_{x}\left(\omega_{c}, \tau\right) \overline{\bar{o}}_{x}\left(\omega_{c}, \tau\right), S_{y y}\left(\omega_{c}\right)=\sum_{\tau=0}^{N-1} \bar{o}_{y}\left(\omega_{c}, \tau\right) \overline{\bar{o}}_{y}\left(\omega_{c}, \tau\right) .
\end{gathered}
$$

In view of the earlier mentioned relation between the FTBPF and the HWT the above formula defines also the frequency dependent HWT coherence Popiński and Kosek (2000).

According to formula (4) we easily obtain Gasquet and Witomski (1999)

$$
\begin{aligned}
S_{x y}\left(\omega_{c}\right) & =\frac{1}{N^{2}} \sum_{\tau=0}^{N-1} \sum_{v=-N / 2+1}^{N / 2} \sum_{\mu=-N / 2+1}^{N / 2} A\left(\omega_{c}, 2 \pi v / N\right) \bar{A}\left(\omega_{c}, 2 \pi \mu / N\right) \tilde{x}(v) \overline{\widetilde{y}}(\mu) \exp (i 2 \pi(v-\mu) \tau / N) \\
& =\frac{1}{N^{2}} \sum_{v=-N / 2+1}^{N / 2} \sum_{\mu=-N / 2+1}^{N / 2} A\left(\omega_{c}, 2 \pi v / N\right) \bar{A}\left(\omega_{c}, 2 \pi \mu / N\right) \tilde{x}(v) \overline{\widetilde{y}}(\mu) \sum_{\tau=0}^{N-1} \exp (i 2 \pi(v-\mu) \tau / N)
\end{aligned}
$$

and since for $v, \mu=-N / 2+1,-N / 2+2, \ldots, N / 2$, we have $-N+1 \leq v-\mu \leq N-1$, the equality

$$
\sum_{\tau=0}^{N-1} \exp (i 2 \pi(v-\mu) \tau / N)=\frac{1-\exp (i 2 \pi(v-\mu))}{1-\exp (i 2 \pi(v-\mu) / N)}=0
$$


holds for $v \neq \mu$, and consequently using the Kronecker delta symbol we can write $\sum_{\tau=0}^{N-1} \exp (i 2 \pi(v-\mu) \tau / N)=N \delta_{v \mu}$, which finally gives

$$
S_{x y}\left(\omega_{c}\right)=\frac{1}{N} \sum_{v=-N / 2+1}^{N / 2}\left|A\left(\omega_{c}, 2 \pi v / N\right)\right|^{2} \tilde{x}(v) \overline{\widetilde{y}}(v)
$$

Analogously, we can obtain the formulae

$$
S_{x x}\left(\omega_{c}\right)=\frac{1}{N} \sum_{v=-N / 2+1}^{N / 2}\left|A\left(\omega_{c}, 2 \pi v / N\right)\right|^{2}|\tilde{x}(v)|^{2} \quad, \quad S_{y y}\left(\omega_{c}\right)=\frac{1}{N} \sum_{v=-N / 2+1}^{N / 2}\left|A\left(\omega_{c}, 2 \pi v / N\right)\right|^{2}|\tilde{y}(v)|^{2} .
$$

If we assume that $A\left(\omega_{c}, \omega\right) \neq 0$ only for $-\pi<\omega_{c}-2 \pi \lambda<\omega<\omega_{c}+2 \pi \lambda<\pi$, the summation in the above formulae comprises the indices $N\left(\omega_{c} / 2 \pi-\lambda\right)<v<N\left(\omega_{c} / 2 \pi+\lambda\right)$, i.e. about $2 N \lambda$ terms.

In order to estimate the magnitude of the coherence statistical error we assume now that $x(n), y(n), n=0,1, \ldots, N-1$, are realizations of independent identically distributed random variables with complex normal distribution $N\left(0, \sigma^{2}\right)$ Brillinger (1975). Then, since $E[x(t) \bar{y}(s)]=0, \quad E[x(t) \bar{x}(s)]=\sigma^{2} \delta_{s t}, \quad E\left[y(t) \bar{y}(s]=\sigma^{2} \delta_{s t}\right.$, for $s, t=0,1, \ldots, N-1$, we see immediately in view of equality (6) that

$$
\begin{gathered}
E[\tilde{x}(v) \overline{\widetilde{y}}(\mu)]=\sum_{t=0}^{N-1} \sum_{s=0}^{N-1} E[x(t) \bar{y}(s)] \exp (i 2 \pi(\mu s-v t) / N)=0, \\
E[\widetilde{x}(v) \overline{\widetilde{x}}(\mu)]=\sum_{t=0}^{N-1} \sum_{s=0}^{N-1} E[x(t) \bar{x}(s)] \exp (i 2 \pi(\mu s-v t) / N)=\sigma^{2} \sum_{t=0}^{N-1} \exp (i 2 \pi t(\mu-v) / N)=\sigma^{2} N \delta_{v \mu}, \\
E[\tilde{y}(v) \overline{\widetilde{y}}(\mu)]=\sum_{t=0}^{N-1} \sum_{s=0}^{N-1} E[y(t) \bar{y}(s)] \exp (i 2 \pi(\mu s-v t) / N)=\sigma^{2} \sum_{t=0}^{N-1} \exp (i 2 \pi t(\mu-v) / N)=\sigma^{2} N \delta_{v \mu}
\end{gathered}
$$

for $v, \mu=-N / 2+1,-N / 2+2, \ldots, N / 2$. In consequence, taking into account (7) and (8)

$$
E\left[S_{x y}\left(\omega_{c}\right)\right]=0 \quad, E\left[S_{x x}\left(\omega_{c}\right)\right]=E\left[S_{y y}\left(\omega_{c}\right)\right]=\sigma^{2} \sum_{v=-N / 2+1}^{N / 2}\left|A\left(\omega_{c}, 2 \pi v / N\right)\right|^{2} .
$$

Putting $\gamma_{x y}\left(\omega_{c}\right)=\gamma_{x y}\left(S_{x x}\left(\omega_{c}\right), S_{y y}\left(\omega_{c}\right), S_{x y}\left(\omega_{c}\right)\right)$, according to (5), and using the standard Taylor formula linearization approach to the function $\gamma_{x y}\left(S_{x x}\left(\omega_{c}\right), S_{y y}\left(\omega_{c}\right), S_{x y}\left(\omega_{c}\right)\right)$ at the point $\left(E\left[S_{x x}\left(\omega_{c}\right)\right], E\left[S_{y y}\left(\omega_{c}\right)\right], E\left[S_{x y}\left(\omega_{c}\right)\right]=0\right)$ we see at once that

$$
\gamma_{x y}\left(\omega_{c}\right) \cong S_{x y}\left(\omega_{c}\right) \frac{\partial \gamma_{x y}\left(\omega_{c}\right)}{\partial S_{x y}\left(\omega_{c}\right)}\left(E\left[S_{x x}\left(\omega_{c}\right)\right], E\left[S_{y y}\left(\omega_{c}\right)\right], E\left[S_{x y}\left(\omega_{c}\right)\right]\right)=\frac{S_{x y}\left(\omega_{c}\right)}{\sqrt{E\left[S_{x x}\left(\omega_{c}\right)\right] E\left[S_{y y}\left(\omega_{c}\right)\right.}},
$$

which yields for coherence magnitude $\kappa_{x y}\left(\omega_{c}\right)=\left|\gamma_{x y}\left(\omega_{c}\right)\right|$,

$$
E \kappa_{x y}^{2}\left(\omega_{c}\right)=E\left|\gamma_{x y}\left(\omega_{c}\right)\right|^{2} \cong \frac{E\left[S_{x y}\left(\omega_{c}\right) \bar{S}_{x y}\left(\omega_{c}\right)\right]}{E\left[S_{x x}\left(\omega_{c}\right)\right] E\left[S_{y y}\left(\omega_{c}\right)\right]} .
$$

Hence, we still have to compute the expectation in the numerator. According to (7), having in view the equalities $E[\tilde{x}(v) \overline{\widetilde{x}}(\mu)]=\sigma^{2} N \delta_{v \mu}, E[\tilde{y}(v) \overline{\widetilde{y}}(\mu)]=\sigma^{2} N \delta_{v \mu}$, we have

$$
\begin{gathered}
E\left[S_{x y}\left(\omega_{c}\right) \bar{S}_{x y}\left(\omega_{c}\right)\right]=\frac{1}{N^{2}} \sum_{v=-N / 2+1}^{N / 2} \sum_{\mu=-N / 2+1}^{N / 2}\left|A\left(\omega_{c}, 2 \pi v / N\right)\right|^{2}\left|A\left(\omega_{c}, 2 \pi \mu / N\right)\right|^{2} E[\widetilde{x}(v) \overline{\widetilde{y}}(v) \overline{\widetilde{x}}(\mu) \tilde{y}(\mu)] \\
=\frac{1}{N^{2}} \sum_{v=-N / 2+1}^{N / 2} \sum_{\mu=-N / 2+1}^{N / 2}\left|A\left(\omega_{c}, 2 \pi v / N\right)\right|^{2}\left|A\left(\omega_{c}, 2 \pi \mu / N\right)\right|^{2} \sigma^{4} N^{2} \delta_{v \mu}=\sigma^{4} \sum_{v=-N / 2+1}^{N / 2}\left|A\left(\omega_{c}, 2 \pi v / N\right)\right|^{4} .
\end{gathered}
$$


Thus, the root mean square error of coherence magnitude $\kappa_{x y}\left(\omega_{c}\right)$ is estimated as follows

$$
\sqrt{E\left|\gamma_{x y}\left(\omega_{c}\right)\right|^{2}} \cong \sqrt{\frac{2 \pi}{N}} \frac{\sqrt{\frac{2 \pi}{N}} \sum_{v=-N / 2+1}^{N / 2}\left|A\left(\omega_{c}, 2 \pi v / N\right)\right|^{4}}{\frac{2 \pi}{N} \sum_{v=-N / 2+1}^{N / 2}\left|A\left(\omega_{c}, 2 \pi v / N\right)\right|^{2}} \cong \sqrt{\frac{2 \pi}{N} \frac{\sqrt{\int_{-\pi}^{\pi}\left|A\left(\omega_{c}, \omega\right)\right|^{4} d \omega}}{\int_{-\pi}^{\pi}\left|A\left(\omega_{c}, \omega\right)\right|^{2} d \omega}}
$$

and since $A\left(\omega_{c}, \omega\right)$ is simply a translation of $A(0, \omega)$ in the frequency domain we finally have

$$
R M S\left[\kappa_{x y}\left(\omega_{c}\right)\right]=\sqrt{E\left|\gamma_{x y}\left(\omega_{c}\right)\right|^{2}} \cong \sqrt{\frac{2 \pi}{N}} \frac{\sqrt{\int_{-\pi}^{\pi}|A(0, \omega)|^{4} d \omega}}{\int_{-\pi}^{\pi}|A(0, \omega)|^{2} d \omega} .
$$

Now, the Schwartz inequality Bremaud (2002) assures that

$$
\int_{-\pi}^{\pi}|A(0, \omega)|^{2} d \omega=\int_{-2 \pi \lambda}^{2 \pi \lambda}|A(0, \omega)|^{2} d \omega \leq \sqrt{4 \pi \lambda}\left(\int_{-2 \pi \lambda}^{2 \pi \lambda}|A(0, \omega)|^{4} d \omega\right)^{1 / 2}=\sqrt{4 \pi \lambda}\left(\int_{-\pi}^{\pi}|A(0, \omega)|^{4} d \omega\right)^{1 / 2},
$$

which together with (10) gives the following approximate lower bound for the RMS error of coherence magnitude $\kappa_{x y}\left(\omega_{c}\right)$,

$$
R M S\left[\kappa_{x y}\left(\omega_{c}\right)\right] \geq \sqrt{\frac{2 \pi}{N}} \frac{1}{\sqrt{4 \pi \lambda}}=\frac{1}{\sqrt{2 N \lambda}} .
$$

The same lower bound can be obtained if we use the Schwartz inequality for sums approximating the integrals in (9). It is easy to see that for the boxcar transmittance function $A_{R}(0, \omega)$ we have equality in the Schwartz inequality, so then we also have approximate equality in (11). As it was already remarked after deriving equations (8) $2 N \lambda$ equals approximately the number of indices $v=-N / 2+1,-N / 2+2, \ldots, N / 2$, that satisfy the conditions $\omega_{c}-2 \pi \lambda<2 \pi \nu / N<\omega_{c}+2 \pi \lambda$. In the case the sampling interval of the analyzed time series $\Delta t \neq 1$ analogous derivation gives us similar formula as in (10) with the factor $\sqrt{2 \pi / N \Delta t}$ instead of $\sqrt{2 \pi / N}$, and then we also have $1 / \sqrt{2 N \lambda \Delta t}$ on the right hand side of (11). In Sejdić et al. (2009) the quotient on the right hand side of (10) is used as a measure of concentration of the filter transmittance function $A(0, \omega)$. As one can clearly see from the derived estimate (10) the statistical error of $\kappa_{x y}\left(\omega_{c}\right)$ does not depend on the central frequency of the filtered oscillation $\omega_{c}$ and this fact was confirmed by the Monte Carlo experiments performed in Popiński and Kosek (2000).

\section{DETERMINATION OF THE OPTIMAL FILTER PASS-BAND WIDTH}

In section 2 it was assumed that the analyzed signal represented by the function $x$ and the considered harmonic wavelet functions $h$ are elements of the Hilbert space $L^{2}(R)$. It is a well-known fact from the theory of Hilbert spaces that the squared norm $\|x-\alpha h\|^{2}$ attains its minimum with respect to scalar $\alpha$ when $\langle x-\alpha h, h\rangle=0$, i.e. when $\alpha h$ is the orthogonal projection of the function $x$ on the linear subspace spanned by the function $h$ Bremaud (2002). Thus, $\alpha_{m}=<x, h>/\|h\|^{2}$ and simple calculation shows that the minimum norm 


$$
\left\|x-\alpha_{m} h\right\|^{2}=\|x\|^{2}-\frac{\left\langle<x, h>\left.\right|^{2}\right.}{\|h\|^{2}} .
$$

Hence, in order to get the best possible approximation in $L^{2}(R)$ of the analyzed signal $x$ by the harmonic wavelet function we should choose functions which maximize the value of $|<x, h>|^{2} /\|h\|^{2}$.

Since in the HWT we compute the values of the scalar products between $x$ and succeeding time domain translations of the wavelet function $h_{\tau}(t)=h(t-\tau)$ with $\left\|h_{\tau}\right\|^{2}=\|h\|^{2}, \tau=0,1, \ldots, N-1$, which depend on the spectral pass-band width parameter $\lambda>0$, we may determine a proper value of $\lambda$ by maximizing the sum of $\left.\left|<x, h_{\tau}\right\rangle\right|^{2} /\|h\|^{2}$. Consequently, according to (2), (5), (8) we obtain for time series data

$$
S(\lambda)=\frac{\sum_{\tau=0}^{N-1}\left|<x, h_{\tau}>\right|^{2}}{\|h\|^{2}} \cong \frac{\sum_{\tau=0}^{N-1}\left|\breve{o}_{x}\left(\omega_{c}, \tau\right)\right|^{2}}{\|h\|^{2}} \cong \frac{\frac{1}{N} \sum_{v=-N / 2+1}^{N / 2}\left|A\left(\omega_{c}, 2 \pi v / N\right)\right|^{2}|\widetilde{x}(v)|^{2}}{\|h\|^{2}} .
$$

Similar measure of optimality was proposed in the work Candès et al. (2008) in the context of detecting oscillatory signals. Let us observe that using the Plancherel identity and discretizing the frequency domain integral by summation with step $2 \pi / N$ gives us an approximation

$$
\|h\|^{2}=\int_{-\infty}^{+\infty}|h(t)|^{2} d t=\frac{1}{2 \pi} \int_{-\infty}^{+\infty}\left|h\left(\omega_{c}, \omega\right)\right|^{2} d \omega=\frac{1}{2 \pi} \int_{-\pi}^{\pi}\left|A\left(\omega_{c}, \omega\right)\right|^{2} d \omega \cong \frac{1}{N} \sum_{v=-N / 2+1}^{N / 2}\left|A\left(\omega_{c}, 2 \pi v / N\right)\right|^{2},
$$

so the value of $S(\lambda)$ is approximated as follows

$$
S(\lambda) \cong \frac{\sum_{v=-N / 2+1}^{N / 2}\left|A\left(\omega_{c}, 2 \pi v / N\right)\right|^{2}|\widetilde{x}(v)|^{2}}{\sum_{v=-N / 2+1}^{N / 2}\left|A\left(\omega_{c}, 2 \pi v / N\right)\right|^{2}},
$$

which is the weighted mean of $|\widetilde{x}(v)|^{2}$ values with weights $\left|A\left(\omega_{c}, 2 \pi v / N\right)\right|^{2}$, $v=-N / 2+1,-N / 2+2, \ldots, N / 2$. The above equation simplifies numerical implementation of selecting the optimal value of $\lambda$ in the case the integral of the function $\left|A\left(\omega_{c}, \omega\right)\right|^{2}$ or equivalently $\|h\|^{2}$ can not be computed analytically. The proposed approach to optimal filter pass-band width determination can be applied in the case of time series in which frequencies of interest can be satisfactorily resolved.

\section{RELATION TO ANALYTIC SIGNAL BASED ON THE HILBERT TRANSFORM}

The aim of this section is to highlight on the problem of filtering oscillations from real-valued signals and show its relationship with forming their complex-valued counterparts using the Hilbert transform. In the case of a square integrable real-valued signal $x(t)=\operatorname{Re}[x(t)]$ the definition of the FTBPF must be slightly modified in order to obtain real-valued oscillations $o_{x}\left(\omega_{c}, t\right)$. Namely, since for real-valued functions $\bar{x}(-\omega)=\overline{\bar{x}}(\omega)$ we must use the modified transmittance function $A_{r}\left(\omega_{c}, \omega\right)=\left[A\left(\omega_{c}, \omega\right)+A\left(-\omega_{c}, \omega\right)\right] / 2$, and then in accordance with definition (1) $C F T\left[o_{x}\left(\omega_{c}, s\right)\right]=A_{r}\left(\omega_{c}, \omega\right) \widehat{x}(\omega)$. Now, we can form the so called analytic complex-valued oscillation from the real-valued oscillation $o_{x}\left(\omega_{c}, t\right)$ putting its Hilbert transform as the imaginary component Gasquet and Witomski (1999) 
where

$$
a_{x}\left(\omega_{c}, t\right)=o_{x}\left(\omega_{c}, t\right)+i H T\left[o_{x}\left(\omega_{c}, s\right)\right](t)
$$

$$
H T[y(s)](t)=\frac{1}{\pi} \int_{-\infty}^{+\infty} \frac{y(s)}{t-s} d s
$$

denotes the Hilbert transform of a function $y(s)\left(y \in L^{2}(R)\right)$, which represents its convolution with the function $g(s)=1 /(\pi s)$ Gasquet and Witomski (1999). The CFT of the Hilbert transform of a signal $y(s)$ has much simpler form, since it is obtained by multiplying $\hat{y}(\omega)$ by $\hat{g}(\omega)=-i \cdot \operatorname{sigh}(\omega)$ Gasquet and Witomski (1999), where the signum function is defined as follows

$$
\operatorname{sign}(\omega)=\left\{\begin{array}{rll}
1 & \text { for } & \omega>0, \\
0 & \text { for } & \omega=0, \\
-1 & \text { for } & \omega<0 .
\end{array}\right.
$$

Consequently, if we assume that $\omega_{c}-2 \pi \lambda>0$, which is not a restrictive condition, then we also have $A_{r}\left(\omega_{c}, \omega\right)=\left[A\left(\omega_{c}, \omega\right)+A\left(-\omega_{c}, \omega\right)\right] / 2=A\left(\omega_{c}, \omega\right) / 2$ for $\omega>0$ and $A_{r}\left(\omega_{c}, 0\right)=0$, hence

$$
C F T\left[a_{x}\left(\omega_{c}, s\right)\right]=(1+\operatorname{sign}(\omega)) A_{r}\left(\omega_{c}, \omega\right) \widehat{x}(\omega)=A\left(\omega_{c}, \omega\right) \widehat{x}(\omega)
$$

Thus, the complex-valued analytic oscillation $a_{x}\left(\omega_{c}, t\right)$ is computed simply by applying the FTBPF definition with the unmodified transmittance function $A\left(\omega_{c}, \omega\right)$ to the real-valued signal $x(t)$. This observation can save the computation time and highlights the properties of the FTBPF technique.

Otherwise, we can first form the analytic complex-valued signal $z(t)=x(t)+i H T[x(s)](t)$ with $\widehat{z}(\omega)=(1+\operatorname{sign}(\omega)) \widehat{x}(\omega)$ and then filter from it an oscillation with central frequency $\omega_{c}$. In order to obtain the analytic complex-valued oscillation $a_{x}\left(\omega_{c}, t\right)$ we must then use the transmittance function $A_{r}\left(\omega_{c}, \omega\right)=\left[A\left(\omega_{c}, \omega\right)+A\left(-\omega_{c}, \omega\right)\right] / 2=A\left(\omega_{c}, \omega\right) / 2$ for $\omega>0$ and $A_{r}\left(\omega_{c}, 0\right)=0$, provided $\omega_{c}-2 \pi \lambda>0$ as before.

\section{DISCUSSION OF RESULTS}

The aspects of the FTBPF technique described in this work were not, as far as the author knows, the subject of any other publication on filtration methods used in time series analysis. Since in the present work we relate the FTBPF technique to the Harmonic Wavelet Transform Newland (1998) it is also connected with research on using different approach to filtering of irregular oscillations, based on the wavelet transform Fabert (2004), Fabert and Schmidt (2003).

It is worth remarking that the way of deriving the estimate of the FTBPF or the HWT coherence statistical error applied in the present work is also applicable in the case of the Morlet Wavelet Transform (MWT) coherence Popiński and Kosek (2000) or the Stockwell STransform coherence Gibson et al. (2006), Stockwell (2007), Stockwell et al. (1996). This follows from the fact that the frequency domain formula for the Continuous Wavelet Transform coefficients with respect to the Morlet wavelet is analogous to the formula on the right hand side of (2), namely Gasquet and Witomski (1999)

$$
W(a, \tau)=\frac{\sqrt{|a|}}{2 \pi} \int_{-\infty}^{+\infty} \bar{x}(\omega) \overline{\bar{\psi}}_{M}(a \omega) \exp (i \tau \omega) d \omega,
$$


where $a \neq 0$ is the period parameter, $\tau \in R$ is the translation parameter of the transform and $\widehat{\psi}_{M}(\omega) \cong \exp \left(-(\omega-2 \pi)^{2} \sigma^{2} / 2\right)$ is the CFT of the Morlet wavelet function $\psi_{M}(t) \cong(\sqrt{2 \pi} \sigma)^{-1} \exp \left(-t^{2} / 2 \sigma^{2}\right) \exp (i 2 \pi t)$ with parameter $\sigma>0$ which controls its decay in time and frequency domain Kulesh et al. (2008), Popiński and Kosek (2000). Derivation analogous to that of section 3 leads to the following estimate of the frequency dependent MWT coherence statistical error

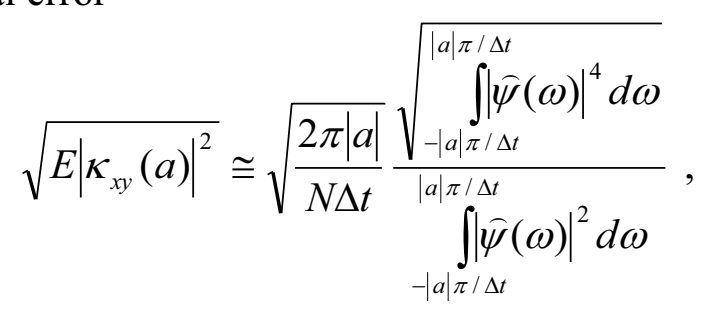

which is similar to the estimate obtained in different way by Van Milligen (1999). The validity of the above formula was confirmed by the Monte Carlo experiments carried out in the work Popiński and Kosek (2000). Taking into account the direct relation between the MWT and the Stockwell S-transform Gibson et al. (2006) it is an easy task to modify the above formula for the statistical error to obtain one that is valid in the case of the STransform.

\section{REFERENCES}

Bremaud P. (2002) Mathematical Principles of Signal Processing: Fourier and Wavelet Analysis, Springer Verlag Inc., New York.

Brillinger D.R. (1975) Time Series - Data Analysis and Theory, Holt, Rinehart and Winston Inc., New York.

Candès E.J., Charlton Ph.R., Helgason H. (2008) Detecting Highly Oscillatory Signals by Chirplet Path Pursuit, Applied and Computational Harmonic Analysis, Vol. 24, No. 1, 1440.

Fabert O. (2004) Effiziente Wavelet Filterung mit hoher Zeit-Frequenz-Auflösung, Veröffentlichungen der Deutschen Geodätischen Kommission, Reihe A - Theoretische Geodäsie, Heft 119, Verlag der Bayerischen Akademie der Wissenschaften, München.

Fabert O., Schmidt M. (2003) Wavelet Filtering with High Time-Frequency Resolution and Effective Numerical Implementation Applied on Polar Motion, Artificial Satellites Journal of Planetary Geodesy, Vol. 38, No. 1, 3-13.

Forbes A.M.G. (1988) Fourier Transform Filtering: A Cautionary Note, Journal of Geophysical Research, Vol. 93, No. C6, 6958-6962.

Gasquet C., Witomski P. (1999) Fourier Analysis and Applications - Filtering, Numerical Computation, Wavelets, Springer Verlag Inc., New York.

Gibson P.C., Lamoureux M.P., and Margrave G.F. (2006) Letter to the Editor: Stockwell and Wavelet Transforms, The Journal of Fourier Analysis and Applications, Vol. 12, Issue 6, 713-721.

Hasan T. (1983) Complex Demodulation: Some Theory and Applications, In Brillinger D.R. and Krishnaiah P.R. (Editors), Handbook of Statistics, Vol. 3 - Time Series in the Frequency Domain, Elsevier Science Publishers, Amsterdam, 125-156.

Hoggar S.D. (2006) Mathematics of Digital Images - Creation, Compression, Restoration, Recognition, Cambridge University Press, Cambridge. 
Kołaczek B. (1992) Variations of Short Periodical Oscillations of Polar Motion with Periods Ranging from 10-140 Days, Report No. 419, Department of Geodetic Science and Surveying, The Ohio State University, Columbus, Ohio, USA.

Kołaczek B. and Kosek W. (1993) Variations of 80-120 Days Oscillations of Polar Motion and Atmospheric Angular Momentum, Proceedings of the $7^{\text {th }}$ International Symposium Geodesy and Physics of the Earth, IAG Symposium No. 112, Potsdam, Germany, 5-10 October 1992, Edited by H. Montag and Ch. Reigber, Springer Verlag, 439-442.

Koopmans L.H. (1974) Spectral Analysis of Time Series, Academic Press, New York.

Kosek W. (1995) Time Variable Band Pass Filter Spectra of Real and Complex-Valued Polar Motion Series, Artificial Satellites - Planetary Geodesy, Vol. 30, No. 1, 27-43.

Kosek W. (2004) Possible Excitation of the Chandler Wobble by Variable Geophysical Annual Cycle, Artificial Satellites - Journal of Planetary Geodesy, Vol. 39, No. 2, 135 145.

Kosek W., Kaczkowski J. (1994) Short Periodic Oscillations in $x$ and $y$ Pole Coordinates of the SLR and VLBI Techniques Detected After Filtering with the Kalman Filter, Proceedings of the $3^{\text {rd }}$ Orlov Conference - Study of the Earth as Planet by Methods of Astronomy, Astrophysics and Geodesy, Odessa, 1992, Main Astronomical Observatory, Kiev, 288-297.

Kosek W., Nastula J., Kołaczek B. (1995) Variability of Polar Motion Oscillations with Periods from 20 to 150 Days in 1979-1991, Bulletin Géodésique, Vol. 69, No. 4, 308-319.

Kosek W., Popiński W. (1999) Comparison of Spectro-Temporal Analysis Methods on Polar Motion and its Atmospheric Excitation, Artificial Satellites - Journal of Planetary Geodesy, Vol. 34, No. 2, 65-75.

Kulesh M., Holschneider M., Diallo M.S. (2008) Geophysical Wavelet Library: Applications of the Continuous Wavelet Transform to the Polarization and Dispersion Analysis of Signals, Computers \& Geosciences, Vol. 34, No. 12, 1732-1752.

Nastula J., Korsun A., Kołaczek B., Kosek W., Hozakowski W. (1993) Variations of the Chandler and Annual Wobbles of Polar Motion in 1846-1988 and their Prediction, Manuscripta Geodaetica, Vol. 18, 131-135.

Newland D.E. (1993) Harmonic Wavelet Analysis, Proceedings of the Royal Society of London, Series A, Vol. 443, 203-225.

Newland D.E. (1994) Harmonic and Musical Wavelets, Proceedings of the Royal Society of London, Series A, Vol. 444, 605-620.

Newland D.E. (1998) Time-Frequency and Time-Scale Signal Analysis by Harmonic Wavelets, In Procházka A., Uhliř J., Rayner P.J., Kingsbury N.G. (Editors), Signal Analysis and Prediction, Birkhäuser, Boston, 3-26.

Pan Ch. (1998) Spectral Ringing Suppression and Optimal Windowing for Attenuation and Q Measurements, Geophysics, Vol. 63, No. 2, 632-636.

Pan Ch. (2001) Gibbs Phenomenon Removal and Digital Filtering Directly through the Fast Fourier Transform, IEEE Transactions on Signal Processing, Vol. 49, No. 2, 444-448.

Park J. (1992) Envelope Estimation for Quasi-Periodic Geophysical Signals in Noise: A Multi-taper Approach, In Walden A.T. and Guttorp P. (Editors), Statistics in the Environmental and Earth Sciences, London, 189-219. 
Popiński W. (1997) On Consistency of Discrete Fourier Analysis of Noisy Time Series, Artificial Satellites - Journal of Planetary Geodesy, Vol. 32, No. 3, 131-142.

Popiński W., Kosek W. (1995) The Fourier Transform Band Pass Filter and its Application for Polar Motion Analysis, Artificial Satellites - Planetary Geodesy, Vol. 30, No. 1, 9-25.

Popiński W., Kosek W. (2000) Comparison of Various Spectro-Temporal Coherence Functions between Polar Motion and Atmospheric Excitation Functions, Artificial Satellites - Journal of Planetary Geodesy, Vol. 35, No. 4, 191-207.

Popiński W., Kosek W. (1999) Spectral Analysis of Sea Surface Topography Observed by TOPEX/POSEIDON Altimetry Using Two-Dimensional Fourier Transform, Report Nr 40 - Space Research Centre PAS, Warsaw 1999.

Press W.H., Flannery B.P., Teukolsky S.A., Vetterling W.T. (1992) Numerical Recipes - The Art of Scientific Computing, Cambridge University Press, Cambridge.

Sejdić E., Djurović I. and Jiang J. (2009) Time-Frequency Feature Representation Using Energy Concentration: An Overview of Recent Advances, Digital Signal Processing, Vol. 19 , Nr. 1, 153-183.

Singleton R.C. (1969) An Algorithm for Computing the Mixed Radix Fast Fourier Transform, IEEE Transactions on Audio and Electroacoustics, Vol. 17, No. 2, 93-103.

Speed T.P. (1985) Some Practical and Statistical Aspects of Filtering and Spectrum Estimation, In Price J. F. (Editor), Fourier Techniques and Applications, Plenum Press, New York, 101-118.

Stockwell R.G. (2007) Why to Use the S-Transform ?, In Fields Institute Communications Vol. 52 - Pseudo-differential Operators: Partial Differential Equations and TimeFrequency Analysis, Edited by L. Rodino, B.-W. Schulze, M.W. Wong, 279-309.

Stockwell R.G., Mansinha L., and Lowe R.P. (1996) Localization of the Complex Spectrum: The S Transform, IEEE Transactions on Signal Processing, Vol. 44, No. 4, 998-1001.

Van Milligen B.Ph. (1999) Wavelets, Non-linearity and Turbulence in Fusion Plasmas, In Van den Berg J.C. (Editor), Wavelets in Physics, Cambridge University Press, Cambridge, 227-262.

Received: 2009-01-08,

Reviewed: 2009-06-07, by W. Kosek,

Accepted: 2009-08-03. 